Slavica

bruxellensia

\section{Slavica bruxellensia}

Revue polyphonique de littérature, culture et histoire

slaves

$1 \mid 2008$

Théâtre

\title{
Une locomotive folle, un feu d'artifice interculturel à la Witkacy
}

\section{Marta Skwara}

Traducteur : Katia Vandenborre et Dorota Walczak

\section{OpenEdition}

\section{Journals}

Édition électronique

URL : http://journals.openedition.org/slavica/63

DOI : 10.4000/slavica.63

ISSN : 2034-6395

\section{Éditeur}

Université libre de Bruxelles - ULB

Référence électronique

Marta Skwara, "Une locomotive folle, un feu d'artifice interculturel à la Witkacy », Slavica bruxellensia [En ligne], 1 | 2008, mis en ligne le 15 octobre 2008, consulté le 01 mai 2019. URL : http:// journals.openedition.org/slavica/63 ; DOI : 10.4000/slavica.63

Ce document a été généré automatiquement le 1 mai 2019.

\section{c)}

Les contenus de Slavica bruxellensia sont mis à disposition selon les termes de la Licence Creative Commons Attribution - Pas d'Utilisation Commerciale - Pas de Modification 3.0 France. 


\title{
Une locomotive folle, un feu d'artifice interculturel à la Witkacy
}

\author{
Marta Skwara \\ Traduction : Katia Vandenborre et Dorota Walczak
}

1 Ecrit par Stanisław Ignacy Witkiewicz en 1923, Une locomotive folle $e^{1}$ est à mes yeux un drame exceptionnel, et ce pour plusieurs raisons. Cela m'a paru évident lors de l'étude de la typologie des personnages ${ }^{2}$ de Witkacy. En effet, dans Une locomotive folle, aucun personnage ne parvient à dominer le texte, ni à lui imposer son action ou son interprétation, au contraire de Gyubal Velleÿtar, de Istvan Szentmichalyi, de JeanMathieu-Charles Lenragey et de Persa Zwierżontkowska ${ }^{3}$. Nous trouverons dans Une locomotive folle des messieurs et des dames hors du commun aux idées grandioses et aux ambitions inassouvies. Pourtant, ce ne sont pas eux qui nourrissent la trame de l'histoire, ils ne mènent pas non plus l'action, que ce soit dans un sens métaphorique ou littéral. C'est la locomotive. Pourquoi Witkacy place-t-il la machine au centre du drame? Est-il vraiment, dans son rire sardonique, contre la mécanisation impitoyablement critiquée de la vie? En mettant la locomotive sur scène, tourne-il en dérision la Forme Pure dans le théâtre ou salue-t-il ironiquement le théâtre naturaliste? Voici les questions que nous allons tenter d'aborder, afin d'approcher le noyau de la conception artistique de Witkacy.

2 Le fait de mettre la locomotive au centre du drame d'une part, et les jeux intertextuels d'autre part, rendent cette pièce exceptionnelle. Dans Une locomotive folle, l'intertextualité acquiert une dimension de parodie de texte littéraire, en particulier de La Bête Humaine d'Emile Zola et des « récits ferroviaires » de Stefan Grabiński. Elle flirte également avec les genres populaires, tels que le western et le film policier. Une locomotive folle joue, en outre, avec les expériences réelles du public : le souvenir des catastrophes ferroviaires en Occident et en Europe de l'Est, la peur de l'inconnu, mais aussi la fascination de la vitesse et de la nouveauté.

3 Witkacy devait probablement se rendre compte de la valeur interculturelle de son texte. Une locomotive folle était destinée, comme quatre autres pièces, à être présentée en Europe ${ }^{4}$. Une des deux traductions françaises (seule une traduction dans cette langue a survécu à la guerre) a été réalisée par Witkacy et sa femme, la version polonaise n'est ainsi, chose 
exceptionnelle, qu'une traduction de la traduction. Une locomotive folle a été traduite dans une bonne dizaine de langues 5 . Tantôt, comme dans l'édition allemande d'un choix de pièces $^{6}$, elle a été le titre identificateur de toute la série, tantôt elle a constitué un événement éditorial à part ${ }^{7}$, tout en restant une représentation scénique importante. La perspective culturelle n'était toutefois pas déterminante, au contraire de l'audace en matière de mise en scène et des possibilités de financement. C'est pourquoi - comme l'a mentionné Alain van Crugten, l'auteur belge de la première adaptation francophone de la Locomotive $^{8}$ après la Deuxième Guerre mondiale - les Français ont rarement mis en scène ce type de pièces, dans lequel une locomotive doit exploser sur scène ${ }^{9}$. Par contre,les Américains ne se sont pas sentis intimidés par la performance (le nombre impressionnant de sept représentations en témoigne); la catastrophe devenait même une sorte d'aimant qui attirait les spectateurs (Witkacy le savait et en faisait part dans ses didascalies ${ }^{10}$ ).

Le dernier aspect remarquable du fonctionnement de la Locomotive repose sur sa dimension interprétative. Il existe trois interprétations de ce drame. La paternité de deux d'entre elles est attribuée à l'Américain Daniel Gerould et au Polonais Jan Błoński, tous deux auteurs de monographies sur Witkacy, la troisième est de Wojciech Tomasik, auteur d'une monographie sur le chemin de fer dans la littérature polonaise.

Il existe bien évidement diverses mentions du texte chez d'autres analystes, mais ce sont les trois interprétations évoquées qui constitueront la base de ma réflexion sur la signification d'Une locomotive folle. Cette base est d'autant plus frappante que, sous la plume de ces trois écrivains, le texte apparaît non seulement sous une autre facette, mais également que la locomotive y acquiert une symbolique et un statut différents. Qu'est donc la locomotive folle de Witkacy? Ses significations ont-elles encore un rapport avec notre monde, bien plus mécanisé ? Est-ce un texte polonais, européen, ou peut être tout simplement universel? Exprime-t-il l'angoisse de Witkacy (et la nôtre) vis-à-vis du progrès fulgurant ou plutôt la fascination de la technique et de ses possibilités illimitées ? Nous amuse-t-il, nous effraie-t-il ou nous ennuie-t-il ? ${ }^{11}$ Une locomotive folle a vu le jour il y a plus de quatre-vingts ans dans un monde sans télévision, ni ordinateur ou téléphone portable.

6 Commençons par la première ambiguïté : l'attitude ambivalente de Witkacy vis-à-vis du chemin de fer, compris comme une manifestation de la culture matérielle de l'humanité contemporaine. Selon moi, elle conditionne tous les doubles sens de la tragicomédie de Witkacy ou plutôt de " sa pièce sans thèse en deux actes avec épilogue ", comme l'indique le sous-titre. Il est impossible d'affirmer de manière univoque que Witkacy admirait et aimait les locomotives d'un amour d'enfant ou qu'il craignait leur puissance dévastatrice. Ses biographes et les chercheurs qui se sont penchés sur son œuvre citent les lettres de son père (des années 1899 et 1900) ${ }^{12}$ pour démontrer la fascination du jeune Witkacy pour les trains. De nombreuses photographies, faites par Stani dans son adolescence, confirment cette thèse. Stanisław Okołowicz et Ewa Franczak ont publié plusieurs de ses photographies de locomotives dans leur album ${ }^{13}$, en y ajoutant des commentaires suggestifs. Leurs idées ont d'ailleurs commencé à se frayer un chemin dans le monde de l'interprétation de l'œuvre de Witkacy. Dans son essai The Crazy Locomotive: Witkacy versus $Z_{0}{ }^{14}$, Wojciech Tomasik les comparait aux études détaillées de portraits, qui étaient caractéristiques des impressionnistes français tels que Manet, Monet et Caillebotte. Daniel Gerould a non seulement décrit les fascinations enfantines et adolescentes de Witkacy, mais il a également signalé ses recherches potentielles dans le domaine du chemin de fer. En effet, Władysław Folkierski, l'un de ses précepteurs, était à la base de la 
construction de la première ligne de train dans les hautes Andes et plus tard responsable de la construction du téléphérique de la station de Zakopane dans les Tatras, la " petite patrie» montagnarde de Witkacy. À ces expériences, Tomasik ajoute les nombreux manuels de chemin de fer, auxquels Witkacy pouvait se référer, dont par exemple: Handbuch für spezielle Eisenbahn Technik. Hrsg. H. von Waldegg (Manuel de technique ferroviaire), Leipzig, 1870. L'engouement personnel et l'intérêt scientifique pour le chemin de fer ne constituent pas les seules expériences culturelles de Witkacy. Et Gerould et Tomasik soulignent qu'en 1906 déjà, le père de Witkacy l'avait informé d'une catastrophe ferroviaire en Russie. Toutefois, seul Gerould voit dans cet événement la source de la pièce écrite plus tard par Witkacy. Citons ses propos :

7 Tomasik a très justement signalé d'autres catastrophes ferroviaires qui ont pu toucher Witkacy. En tant qu'officier de l'Armée du tsar et puis comme commissaire de l'Armée Rouge, il a vécu à la fois la sauvagerie de la révolution bolchevique et les incroyables catastrophes de chemins de fer qui avaient lieu en Russie. Ici aussi Gerould a remarqué un lien entre ces expériences sanglantes et la création ultérieure de Witkacy. Pour ce faire, il s'appuie notamment sur l'opinion d'Atanazy Bazakbale, le héros du roman Adieu à l'automne (1927), qui remettait en question le sens de la résistance aux terribles changements égalitaires qui touchaient les sociétés contemporaines. En effet, la tentative de les renverser allait se passer « comme si quelqu'un mettait un bâton joliment sculpté dans les roues de la locomotive, en essayant de l'arrêter ${ }^{16}$.

8 Tomasik suggère également que, au terme de son séjour en Russie, à la fin de l'année 1917, Witkacy a pu lire dans la presse russe des articles sur les catastrophes européennes tristement célèbres, comme celle survenue à Modane le 12 décembre 1917. À cause de l'excès de vitesse et de la surcharge, plus de 800 soldats français avaient perdu la vie dans le train qui avait déraillé. Il est possible, toujours d'après Tomasik, que Witkacy ait eu connaissance de la spectaculaire catastrophe de la gare Montparnasse, alors connue de tous, entre autre grâce à la célèbre photographie (sur)réaliste représentant la locomotive suspendue dans le vide. Cette catastrophe a eu lieu 5 ans après la publication de La bête humaine de Zola et a ébranlé son optimisme. Symbole du progrès et du triomphe de la technique sur une humanité régie par les lois de l'atavisme, la locomotive s'est transformée en un instrument symbolique d'autodestruction ${ }^{17}$.

Nous pouvons dire que, d'une part, Witkacy était fasciné par les locomotives, ce que confirment ses dessins et ses photographies, et que, d'autre part, il pouvait les percevoir comme des instruments de destruction et de triomphe de la machine sur l'homme. Au premier coup d'œil, ces deux positions contradictoires semblent difficilement conciliables. Non seulement les interprétations données par Gerould et Tomasik ne résolvent pas le problème mais renforcent encore le nombre d'antinomies. Cependant, Gerould démontre que Witkacy a créé une pièce noire, «le drame du siècle mécanisé ». Dans cette pièce, la folie a déjà été mécanisée et l'asile, habité par une galerie d'individualistes anachroniques, a été mis sur roues. Ce train file à toute vitesse vers l'apocalypse. À la fin, Gerould dit ceci : «Il ne reste que des débris immobiles de la personnalité brisée de l'homme et de la destruction de la machine, fruit de son savoir technique. » [Ger 312]

Pour Tomasik, Une locomotive folle est «à n'en pas douter le plus somptueux des documents témoignant de l'enthousiasme de Witkacy pour les trains » [Tom 158], c'est un document qui est né de « la protestation contre les conceptions esthétiques qui mettent sur le même pied d'égalité la science et la pratique artistique, la réception de l'œuvre et la connaissance de la réalité » [Tom 176]. Dans la conclusion de son interprétation, il 
affirme : « La locomotive de Witkacy devait, comme la drogue, transporter le spectateur dans "un monde complètement différent". » [Tom 176]

11 Le troisième interprétateur, Jan Błoński (qui ignore totalement l'expérience culturelle de Witkacy liée au transport ferroviaire), affirme que la locomotive est dans cette pièce " une machine dangereuse mais plutôt pacifique ${ }^{18}$ et que le public s'attend plutôt à des complications érotiques entre les quatre héros (deux hommes et deux femmes), sans vraiment se concentrer sur la locomotive. L'action, selon Błoński, se résume à « des démarches visiblement vaines, et donc comiques, des passagers (...) » ${ }^{19}$. La catastrophe finale, si tragique dans la lecture de Gerould et un peu psychédélique dans celle de Tomasik, est, aux yeux de Błoński, surtout comique puisque c'est la parodie qui y domine. La pièce est perçue comme une parodie du théâtre des pressentiments et des rêves de Maeterlinck : on aurait pu éviter la catastrophe si Jeanne Gegon, la folle épouse du GardeVoie, n'avait pas obéi à un fantôme qui, dans son rêve, lui avait ordonné de couper le fil téléphonique.

Les trois interprétateurs proposent trois approches différentes du texte et de son héroïne en titre: la locomotive. Je vais analyser les points les plus importants de leurs interprétations afin de tenter de répondre aux questions posées au début de cet article. Je commencerai par rappeler les idées de Witkacy, ceci étant nécessaire pour saisir le contexte. L'auteur d'Une locomotive folle pense que l'art issu du réalisme, et surtout le théâtre naturaliste, n'ont pas de raison d'être. Conformément à sa théorie de la Forme Pure, dans les œuvres d'art contemporain, il n'y a plus de place pour la probabilité psychologique puisque le but de la pièce ne consiste pas en la représentation d'une réalité possible, mais elle doit rendre accessible au spectateur l'expérience du sentiment métaphysique. Cette opinion était étroitement liée à la vision qu'avait Witkacy de l'humanité, telle qu'il l'a présentée en 1919, après son retour de Russie. Sa principale thèse historio-sophique, exposée dans le traité Nouvelles Formes dans la peinture et les malentendus qui en résultent, affirmait que, depuis la Révolution Française, nous observons une ascension des masses dans la hiérarchie sociale et une démocratisation de la culture, mais qu'en même temps la puissance créatrice des individus disparaît. L'humanité s'achemine vers "le bonheur pour tous", mais le prix à payer pour ce bonheur sera une certaine autosatisfaction et le plaisir écervelé privant l'être humain de sa condition tragique, dans laquelle se reflète sa dignité d'antan. Aujourd'hui, comme l'écrivait Witkacy dans les années 1920, l'homme n'est pas capable d'assouvir ses sentiments métaphysiques par la religion ou la philosophie; il ne reste plus que l'art comme moyen d'expression du « sentiment métaphysique de l'étrangeté de l'existence ». Pourtant, l'art, tout comme la pensée et la culture humaines, subissent une continuelle standardisation et un nivellement vers le bas parce que leur diversité a cessé d'être fonctionnelle. La production, en ce compris la production culturelle, devient un but en soi, mais, en raison de sa mécanisation et de sa monotonie, elle a de moins en moins de choses en commun avec la création. Cela mène à une crise profonde de la culture; l'art meurt car les sentiments métaphysiques qui l'ont inspirée par le passé disparaissent et les médias de masse la réduisent au rôle de divertissement populaire. Tout ce que l'artiste peut faire, c'est essayer d'atteindre et de maintenir le plus haut niveau d'expérience et de compréhension du monde, tout en accordant un intérêt (auto-)dérisoire aux derniers représentants de la race des individualistes ${ }^{20}$, menacés d'être condamnés à la médiocrité dans le monde. Souvent traités de fous ou d'idiots, grotesques dans leur apparence et leur comportement, ils ne parviennent pas à se retrouver dans le monde de la mécanisation et 
de la grisaille, où l'individualité a remplacé le fétiche qu'était la communauté. Dans un tel monde, la machine, et donc la locomotive, ne devraient être que le symbole d'une bête mécanisation, encore un engin rendant la vie humaine plus facile, plus rapide et plus irréfléchie. Or ce n'est pas le cas.

Regardons de plus près Une locomotive folle, une pièce "sans thèse ", comme l'indique avec provocation son auteur dans le sous-titre, en soulignant en exergue l'absurdité de son œuvre $^{21}$. Les deux protagonistes semblent être le machiniste et le chauffeur de chaudière, mais ils s'avèrent être deux fameux criminels. Ils sont accompagnés de deux partenaires : la plus âgée étant la femme du machiniste, qui a par le passé participé à ses crimes, la plus jeune étant la fiancée du chauffeur de chaudière. Witkacy les place tous les quatre dans une locomotive lancée à toute vitesse, et qui continue à accélérer encore proportionnellement au déroulement de l'action. Bien que les protagonistes n'aient pas de plan concret au départ, trois d'entre eux décident de foncer dans le train qui arrive de face. La femme qui avait des doutes quant à cette décision est poussée hors du train et s'écrase "contre une pompe ", ce que confirme son mari Zygfryd Tengier alias Trefaldi ${ }^{22}$ avec une satisfaction évidente. Le trio de fous se prépare à entrer en collision avec le train $\mathrm{n}^{\circ} 50$ au carrefour appelé Dumbell-Junction à l'américaine ${ }^{23}$. Entre-temps, les passagers essayent en vain d'éviter la catastrophe. Il semble donc que la pièce puisse être vue sous au moins trois angles : comme la parodie d'un drame naturaliste, comme une imitation de l'art populaire (surtout le cinéma) ou comme la parabole de la mécanisation de la vie, qui constitue un des principaux maillons de la réflexion historiosophique de Witkacy. À chacun de ces niveaux, la locomotive doit remplir un rôle important, mais trompeur. On pourrait se demander pourquoi la locomotive a été représentée de manière naturaliste dans une parodie de l'art naturaliste? Pourquoi est-ce justement la locomotive qui a été choisie comme indicateur de la convention de l'art populaire? Dans quel sens peut-elle être incorporée à un discours-clé sur l'avenir de l'humanité? Puisque la pièce est hautement ironique et incroyablement comique, de telles questions ne trouvent pas facilement de réponses et les interprétateurs de Witkacy ne facilitent pas la tâche aux lecteurs ou aux spectateurs. Błoński ne peut se retenir de souligner le comique de la langue, en citant les jeux de mots, comme ceux qui apparaissent dans le dialogue entre la femme du machiniste et l'amante du chauffeur de chaudière :

(interprétation de Błoński, Une locomotive folle est surtout une pièce très comique admirablement composée, dans laquelle la locomotive ne joue pas le rôle le plus important. Gerould, lui, se concentre sur les éléments tragiques et prophétiques de la pièce et n'accorde pas une attention particulière à l'aspect comique (en vérité, il ne remarque pas certains jeux de langage, ce que démontre sa traduction de la Locomotive), insistant surtout sur "le voyage destructeur en locomotive folle». Dans l'analyse des éléments parodiés de cette pièce, Tomasik ne parvient pas à résoudre la principale contradiction. Elle repose sur la tension grandissante entre les mécanismes de la parodie, utilisés par Witkacy dans le but de dévoiler les diverses conventions « ferroviaires » dans la vie et dans l'art, et la fascination suggérée pour les trains. Or, parodier ce par quoi nous sommes fascinés n'est-il pas contradictoire?

Afin de résoudre les contradictions, je commencerai par la comparaison de la représentation de la locomotive dans le roman naturaliste et dans Une locomotive folle. En citant Zola et les analyses de son œuvre, surtout celles de Marco Barola, Tomasik affirme clairement que la scène finale de La Bête Humaine constitue une preuve supplémentaire de l'amour de Zola pour le progrès. Le titre le démontre : la bête se rapporte à l'homme et 
non à la machine. La thèse du roman naturaliste repose sur l'éloge du progrès, qui, comme la locomotive, fait avancer l'humanité, indépendamment de l'impulsion ou du lourd héritage génétique. Ainsi, dans la dernière scène du roman de Zola, le train sans conducteur et bondé de soldats ivres avance à toute allureet apparaît non comme le symbole d'une catastrophe inévitable de l'humanité abasourdie, mais comme le signe du triomphe du progrès sur la nature atavique de l'homme, sur la «bête humaine ». Witkacy semble parodier le champ de significations propres à Zola, en appelant sa locomotive: " la bête ", « la bête de fer ", « la bête filant à toute vitesse ", « la machine enragée », « le petit tas de ferraille ", ce qui démontrerait que son attitude vis-à-vis de la locomotive est différente, comme s'il n'en percevait ni la beauté ni l'optimisme. La locomotive est présentée de la sorte dans les didascalies de son premier acte :

Il dépeint plutôt un objet effrayant et gigantesque qu'un objet d'admiration. Le fait que le modèle de la locomotive put être alors "encore inconnu » suggère une part de fantaisie ou du moins une distance par rapport à la convention naturaliste. Mis à part ce seul élément, la scène devait être aménagée de manière naturaliste et son naturalisme devait renforcer et dévoiler l'appareil cinéma-tographique :

Ainsi, le spectateur ou le lecteur imaginatif fait face à une immense machine dangereusement vivante. Dans son introduction à l'édition américaine d'Une locomotive folle, Gerouldécrit ainsi : «Witkiewicz puts the machine on the stage, turns it on, and lets us see where it will lead $»^{24}$ (trad. : Witkiewicz met la machine sur scène, met le contact et nous laisse voir où elle nous conduit). Dans la phrase suivante de son commentaire, l'interprétation se substitue à la description : "The machine is a self-destructive machine - it blows itself up» (trad.: La machine est une machine auto-destructrice : elle va exploser d'elle-même). En effet, il est très difficile de trouver dans le texte des éléments qui prouvent les propriétés destructrices de la machine. Il semble qu'elle éclate tout simplement en raison d'un excès de vitesse, provoqué par les deux célèbres criminels. En arrivant à lancer la machine à la vitesse invraisemblable de $130 \mathrm{~km}$ à l'heure (n'oublions pas que la pièce a été écrite il y a plus de 80 ans!), l'un d'eux, Tengier alias Trefaldi, proclame :

18 Julie, la plus jeune des femmes, follement amoureuse des deux hommes à la fois, décrit son état anormal d'une manière encore plus forte, en évoquant la catégorie, caractéristique de l'œuvre de Witkacy, de l'inassouvissement de la réalité :

L'événement mineur que constitue la panne dans la machine n'est pas particulièrement important. Et peut-être ce ne sont que des divagations de fous puisque Nicolas Wojtaszek, alias Travaillac, affirme qu'il faudra utiliser deux leviers pour arrêter le train parce que «le régulateur est en panne ». De plus, cette explication bizarrement réaliste de la catastrophe a curieusement disparu de la traduction de Gerould. Nous ne saurons donc pas grand-chose de ce régulateur défaillant ${ }^{25}$, bien que, dans l'introduction de la pièce, la propriété « autodestructrice » de cette machine ne soit pas si évidente.

20 Le jeu de Witkacy avec les conventions naturalistes devient de plus en plus astucieux. Walery Kapuscinski («employé de banque et secrètement adepte du formisme en peinture »), qui a héroïquement sauté sur le train filant à toute vitesse afin d'éviter la catastrophe, est accueilli par les cris désespérés de Julie : « Rien ne peut être beau en ce monde ! Le bétail humain s'en mêle toujours et gâche tout ! ! [LF 117]

Dans la version française d'Une locomotive folle,à la place de "ludzkie bydlę », nous trouverons le bétail humain, beaucoup plus proche de la bête humaine de Zola que human 
animal,dont Gerould se sert dans sa traduction. Si nous lisons dans cette formulation une allusion au texte de Zola, l'image du train comme celle de la bête devient à nouveau ambiguë puisque les gens sont, à la manière de Zola, qualifiés de bêtes ${ }^{26}$.

Qui est donc ce monstre meurtrier : la locomotive qui anéantit l'homme ou l'homme qui fait échouer l'aventure folle? Ou peut-être est-ce Travaillac, l'auteur des "plus beaux meurtres ", qui incarne cette bête? Et si l'on peut ressentir ici l'élément de fascination (caractéristique des émerveillements de Witkacy pour les grandes gens, même si leur grandeur se manifeste dans le crime), il est difficile de trouver une telle fascination dans les descriptions de la locomotive. En signalant l'utilisation par Witkacy d'un vocabulaire spécialisé et de termes techniques, Tomasik souligne ses connaissances professionnelles. Les informations concernant la vitesse grandissante ou les noms des différents appareils devaient selon les intentions de Witkacy «entraver l'interprétation mimétique » [Tom 168]. Il semble pourtant que nous soyons confrontés à deux phénomènes différents. D'une part, les paramètres donnés de façon réaliste et logique (atteignant le record de vitesse de l'époque qui était de $130 \mathrm{~km}$ à l'heure ${ }^{27}$ ) soulignent l'engouement finalement mimétique pour le voyage fou, ce qui peut être interprété comme la fascination de la vitesse (Trefaldi s'adressant à Julie : « Mais tu le vois bien, je conti-nue à filer à 130 à l'heure et tu grognes encore! Oh quel inassouvis-sement !» [LF 117]). D'un autre côté, l'utilisation de termes techniques, dans la plupart des cas compréhensibles pour le public, peut effectivement constituer une preuve de parodie de la convention naturaliste (cela concerne aussi d'autres procédés, comme les fameuses descriptions de héros par Witkacy ${ }^{28}$ ). Il est surprenant que les trois exemples cités par Tomasik (la connaissance des moteursà vapeur [compound], les freins pneumatiques Westinghouse, ou le système de dispatching de Heisinger von Waldeck) ne soient pas repris dans son analyse de la parodie et de la dérision au sujet de la convention naturaliste. Ils se rapportent plutôt à l'introduction de Tomasik, qui donne des preuves des passions ferroviaires de Witkacy [Tom 159-160]. Il faut probablement reconnaître que Witkacy a en partie fait la même chose que Zola: après avoir mené une étude approfondie et passionnée dans le domaine des trains, il a introduit des termes spécialisés dans son œuvre littéraire. Il ne fait pas toujours cela dans un but parodique : parfois, il transpose tout simplement sa fascination sur les héros de la pièce. Vers la fin de ce drame, on demande à un des voyageurs de troisième classe, le voleur menotté qui se dit mécanicien, d'aider à arrêter le train, ce qu'il fait facilement :

Il est difficile d'y voir une parodie. On y voit plutôt de la nostalgie et de l'admiration. L'habileté de l'ex-machiniste et la perfection du système de Heisinger von Waldeck sont vains: le $n^{\circ} 50$ fonce à pleine vapeur en direction de la «locomotive folle» et la catastrophe est inévitable. C'est ici que se cache le secret de l'interprétation de la fin du drame et il en est de même dans la traduction. Dans la traduction de Gerould, «a violent crack is heard inside the machine " (trad. : on entend un fracas soudain à l'intérieur de la machine), alors que, dans les versions française et polonaise, il n'y a rien de soudain, on entend tout simplement un «fracas dans la machine» / «trzask wewnĄtrz maszyny » : il peut s'agir du bruit de la coulisse, ce qui n'inquiète personne. Dans la version française ("La vapeur cache tout et on voit la machine qui éclate en morceaux »), tout comme dans la polonaise, il n'y a pas de lien de cause à effet entre la vapeur qui couvre tout et la machine qui éclate en morceaux, mais ce lien existe dans la traduction de Gerould. Nous y lisons: "a terrible crash and din are heard. Steam covers everything, as the engine burst into fragments ${ }^{29}$ (trad.: On entend un horrible fracas, du vacarme. La fumée couvre tout parce que l'engin éclate en mille morceaux). L'utili-sation $\mathrm{du}$ « as » (parce que, puisque) au lieu 
de « on voit » justifie finalement la puissance autodestructrice de la machine, soulignée par Gerould dans son introduction au drame. Mais cette justification n'est pas valable au théâtre puisque le spectateur ne connaît ni les didascalies, ni les remarques de l'auteur à propos de son texte. Il ne peut donc pas deviner que la machine éclate d'elle-même puisque ni les dialogues ni l'épilogue n'en parlent. À vrai dire, personne ne sait ce qui s'est passé ; même le conducteur s'écrie, bras ouverts : « MMMmmysttttèrrrrrrre !! ! » [LF 123] Bien sûr, à la fin, la machine éclate, ce qui accentue le sens catastrophiste de la pièce et écarte l'interprétation basée sur la fascination de Witkacy. En insistant sur le fait que la locomotive de Witkacy devait transporter les spectateurs dans un autre monde, Tomasik n'accorde pas une grande attention à la catastrophe finale. À la lumière du massacre final, cet « autre monde » aurait sonné trop naturaliste (Trefaldi, «le plus grand criminel du monde entier» [LF 126] meurt en communion avec la machine en ayant «les tripes à l'air » [LF 124] tandis que le régulateur lui est entré « d'au moins trente centimètres » [LF 124] dans le ventre). Sinon, ce monde aurait une signification trop absurde (Julie commente la catastrophe ainsi : «Il ne peut rien exister de beau dans la vie! Tout est de la cochonnerie! Tout ce qu'il y a de beau est fini une fois pour toutes! Assez! Assassinezmoi ! " [LF 123-124]). La réaction du troisième héros, le chauffeur de chaudière, réduit la catastrophe finale à une grande déception et provoque l'abandon des plans envisagés : «Tout n'a pas marché comme il fallait. Après tout cela, vraiment, je ne désire que la tranquillité.» [LF 124] On peut mourir ou rester dans un monde gris, attendre et regarder : «(...) les trains qui passent, qui vont quelque part et emmènent tant de gens, tant de gens » [LF 125], comme le dit la femme névrosée du Garde-Voie, expliquant ainsi pourquoi elle a coupé les fils. C'était sa protestation contre la grisaille et un quotidien minable : elle ne voulait plus n'être qu'un spectateur dépassé par les trains.

Les spectateurs de Witkacy se sentent-ils emportés par la locomotive (comme le veut Tomasik) ou sont-ils plutôt laissés en retrait (comme le préfère Gerould)? J'aurais répliqué à Gerould que la catastrophe finale ne doit pas forcément être une vengeance de la machine sur le modèle de la révolte moderne. Peut-être est-elle tout simplement la seule solution scénique possible. Même le titan du théâtre qu'était Witkiewicz n'a pas pu exiger des metteurs en scène qu'ils représentent une collision frontale de deux locomotives sur scène (néanmoins, Trefaldi a une vision si claire qu'il va vers cette collision puisque «ce crétin de train de marchandise ne marcherait jamais à 130 en arrière » [LF 118]). Par contre, à la place de cet événement si attendu, les spectateurs assistent à la mystérieuse dégradation de la locomotive. Dans l'épilogue, le Docteur Wasnicki commente les plans d'arrestation de Trefaldi: "Le public ne se doutait de rien. » [LF 126] Mais le public a ici un double sens : il peut signifier « l'opinion publique » ou «le public du théâtre ». Ce n'est d'ailleurs pas le seul jeu de mot. Avant cela, nous avons eu à faire à la coulisse du système de freinage (ce qui n'est pas visible dans la traduction de Gerould). Or, la « coulisse » (comme le souligne Tomasik) a de nouveau un double sens : technique et théâtral. Il en est de même pour la " comédie ", utilisée dans le sens de "vie absurde ", derrière laquelle se cache le nom d'un genre scénique, ou encore pour le « machiniste » qui manie non seulement les locomotives mais aussi la machinerie théâtrale. Cela échappe de nouveau à Gerould, qui appelle le machiniste «a locomotive engineer ». En plus, de la scène nous pouvons entendre la déclaration suivante: «Pour l'amour de Dieu, Monsieur le chef de train, ne perdons pas de temps à discuter ! Ceci n'est pas une représentation de théâtre ! » [LF 119] 
25 Ajoutons que le public est justement assis dans un théâtre ou qu'il lit la pièce en pensant qu'elle a été écrite pour le théâtre. Et Trefaldi commente ainsi la situation du «trio de fous » sur la locomotive qui file : «Sur ce sujet on ne peut rien dire d'intéressant, sauf les carabistouilles ordinaires, avec lesquelles les auteurs dramatiques peu habiles remplissent le vide de leurs pièces. Alors attendons !» [LF 120]

26 La convention naturaliste n'a pas été la seule à vaciller sous le poids de l'ironie : il en est de même pour les conventions cinématographiques ${ }^{30}$. Les paroles de Julie valent la peine d'être rappelées dans ce contexte : «J'ai toujours rêvé de quelque chose d'extraordinaire, de quelque chose comme au cinéma !! » [LF 112] La locomotive est utilisée surtout comme un objet et un lieu d'action tandis que chez Witkacy elle est aussi l'héroïne de l'action. Evidemment, cela peut poser des problèmes d'interprétation. L'anthropomorphisation de la locomotive se déroule sur différents plans : dans le titre, dans les expressions utilisées dans les didascalies (comme par exemple : «La machine souffle de plus en plus vite » [LF 106]) ou encore dans les tournures qui suggèrent que la locomotive est une bête. Parfois même, cette bête interrompt les répliques colériques des héros, par exemple quand Julie prononce ses déclamations exaltées :

L'identification de l'homme à la machine relève de l'anthropomorphisation, tout comme la mort que le héros s'est donnée de "sa propre main", agonisant cependant avec un "régulateur dans le ventre $»^{31}$. En réunissant tous ces éléments, on peut soutenir l'interprétation de Gerould qui comprend la locomotive comme « la machine faisant tout éclater en l'air ", capable comme un être humain de décider de ses actes et quand les poser. Nous pouvons pourtant voir l'anthropomorphisation d'une manière un peu différente.

En interprétant le texte de Zola, Tomasik signale le fait évident que, dans son roman, il y a deux machines : l'une a seulement un numéro (608), l'autre un prénom féminin (Lisette). Ensemble elles symbolisent deux stades du procès de la familiarisation des machines. Chaque machine doit, d'après le texte de Zola, être domptée comme une jeune jument. Alors seulement elle sert le progrès, grâce auquel tout le monde s'achemine vers le pays du bonheur (voir [Tom 164]). Or, Witkacy ne faisait pas confiance aux utopies : il voyait en elles une menace contre l'humanité plutôt que la résolution de dilemmes existentiels. Peut-être voulait-il montrer l'impossibilité de domestiquer la machine qui, laissée à ellemême, sera toujours une bête sauvage qui tue des gens: littéralement et métaphoriquement. Dans ce sens-là, il est en désaccord avec l'optimisme de Zola ainsi qu'avec ses conventions littéraires. Jusqu'ici il n'y aurait donc pas de grande contradiction entre les deux interprétations. Ajoutons que la fascination des progrès techniques ne va pas de pair avec le pessimisme de Witkacy en ce qui concerne la civilisation. Il semble néanmoins qu'iln'ait pas été aussi fasciné par les machines que Zola ou les futuristes, mais plutôt par les êtres humains capables d'inventer et de donner vie à de magnifiques trouvailles techniques. Il suffit de rappeler que Witkacy a, par admiration pour les différentes parties de la locomotive, utilisé des noms de constructeurs : Heisinger von Waldeck et Westinghouse. Dans ce dernier cas, le mépris pour la bête machine ainsi que le respect pour l'homme et la confiance en celui-ci trouvent leur expression dans la stylistique du texte. Quand Nicolas ordonne: «Fermez ce maudit régulateur et faites fonctionner les freins, c'est-à-dire laissez agir Monsieur Westinghouse ${ }^{32}$, car nous pourrions dérailler dans ce tournant ", alors «le souffle de la machine s'arrête » [LF 107] et elle réagit docilement. 
29 Il est manifeste que Witkacy admirait l'élan des locomotives comme un des moyens permettant à l'homme contemporain et surtout à l'artiste, si limité par le développement social, de pénétrer dans un autre monde, un peu comme le peyotl, dont nous trouvons un célèbre éloge dans Drogues (1932). Selon Tomasik [Tom 159], ses photographies et dessins plus tardifs en témoignent. Le ton un peu enfantin de ces dessins montre que l'auteur avait une conscience aiguë de sa fascination ferroviaire, à la fois enfantine et violente. Ce n'est pas pour rien que, comme l'indique Gerould [Ger 304], les deux principaux héros et meurtriers veulent jouer à Robinson et Vendredi. Par ailleurs, une des épigraphes provient d'une lecture de jeunesse de Witkacy: L'île au trésor. La personnification de la locomotive, vue du point de vue du jouet pour enfant, peut être interprétée comme un élément de plus dans l'établissement d'une distance entre l'art et la réalité. Mais elle se situe aussi entre, d'une part, l'indomptable vision enfantine de Witkacy (et la nôtre) et, d'autre part, la capacité adulte, pesée et raisonnée (mais aussi insuffisante) de perception logique du monde. Pourtant, d'après Witkacy, le véritable danger ne vient pas tant du fait de jouer naïvement " aux trains ", par rapport auquel nous pouvons nous distancier à tout moment, mais plutôt de la foi naïve en la mécanisation, qui est censée résoudre nos problèmes humains. C'est probablement pour cette raison que Witkacy diabolise quelque peu sa locomotive, qui doit servir « d'épouvantail » au progrès. Pour Witkacy, la foi en l'art qui glorifie les machines était dangereuse elle aussi, d'où ses parodies des conventions naturalistes et futuristes ainsi que de la culture populaire avec ses excitantes aventures ferroviaires ${ }^{33}$.

30 La fascination des pouvoirs créateurs de l'homme, et pas seulement de ses œuvres, n'est pas en contradiction avec la condamnation de la mécanisation comprise comme un but en soi. Dans le premier cas, l'homme est le sujet de l'action (ce que Witkacy exige toujours de l'Existence Particulière) ; dans le deuxième, il est réduit à l'objet. Ainsi, la locomotive de Witkacy devient le rêve d'une humanité qui atteint le sommet de sa créativité. Dans ce sens, je donne raison à Tomasik qui écrit que «comme la drogue, elle transporte le spectateur dans un autre monde » [Tom 176], mais aussi à Gerould qui parle des désirs humains de miracles techniques qui, au final, tuent cette humanité. Il n'y a rien d'étonnant à ce que les deux interprétateurs soulignent différents aspects de l'art. Le premier s'intéresse surtout aux conventions de représentation du train dans la littérature. Le deuxième interprétateur, lui, se penche sur l'ensemble de l'œuvre de Witkacy et en souligne surtout le message catastrophiste. La pièce elle-même repose bien évidement sur des prémisses contradictoires, tout comme l'ensemble de l'écriture de Witkacy, qui, non sans raison, est le plus souvent qualifiée de grotesque, esthétique qui se nourrit de contradictions. Nous devrions nous pencher non pas sur la question de l'engouement de Witkacy pour les locomotives ou de la peur qu'il en a, mais plutôt sur la manière et le but de l'utilisation de ces deux expériences contradictoires.

31 C'est pour cette raison que je mettrai la locomotive au premier plan, contrairement à Błoński qui préfère se concentrer sur l'action du drame. Remarquons que le train filant à toute vitesse au milieu de nulle part éveille aussi d'autres associations dans notre mémoire culturelle. Et puisque les héros qui voyagent dans le but de vivre une expérience existentielle, un moment d'extase qui change toute la vie, sont souvent qualifiés de "fous ", la locomotive semble constituer une version contemporaine du "bateau des fous ». Tengier mentionne même au début du texte que pour leurs besoins « un navire, ça irait mieux, mais je ne supporte pas l'eau ni les problèmes qui s'y rattachent... ${ }^{34}$ [LF 104] La locomotive incarne la traditionnelle symbolique à double sens du stultifera navis : la 
barque des idiots etle bateau des fous sont à la fois une fuite déraisonnable des idiots et une recherche des follement courageux ${ }^{35}$, dont les buts et les intentions se croisent et se superposent souvent dans la perspective culturelle.

Entreprise au nom de Dieu, la quête du savoir le plus grand, frôlant les limites de la folie, pouvait mener soit à la renaissance symbolique soit à la perte définitive. Witkacy semble suggérer que rien n'a changé au cours des siècles dans la nature humaine : nous sommes toujours à la recherche de l'illumination au bord de l'annihilation ${ }^{36}$. Le changement du bateau en locomotive ${ }^{37}$, des prières en injures, des voiles blanches en locomotive noire rend ce voyage plus diabolique et pourtant pas moins humain. Car ce n'est pas la locomotive qui est devenue folle (de la même manière ni le bateau ni la barque n'étaient fous), mais les gens avec leurs désirs et leurs souhaits jamais assouvis. Indépendamment de la vitesse et de la fluidité de ce voyage, le dilemme restera le même et aucune solution technique ne pourra le résoudre. Les voies de chemin de fer rendent encore plus parlante la parabole de Witkacy sur l'illusion du progrès sauveur de l'humanité, sur l'illusion de toute utopie humaine et surtout technique : les rails ne mènent que là où ils ont été construits. Tengier le sait avant d'entamersonvoyagefou: "Où ils [les rails] nous conduiront finalement, nous ne le savons que trop bien. » [LF 104]

Avec toutes ces situations comiques, ces allusions qui s'enchainent, ces provocations antimimétiques, ces dérisions et autodérisions, la question, simple à en être provocante, que pose le machiniste : «Faut-il regarder de la machine vers la terre ou de la terre vers la locomotive?» [LF 105] dépasse la frontière de l'absurde. La réponse de Witkacy est claire: toujours de la terre vers la locomotive, du point de vue de l'homme vers la machine car, si nous faisons le contraire, nous nous casserons la figure. En faisant de la locomotive son héros principal, Witkacy nous invite à jouer culturellement: avec l'enfance, avec nos lectures et avec les films que nous avons en mémoire. Le tout pour résoudre la devinette : qui est le héros? Néanmoins, Witkacy se sert de notre mémoire culturelle pour construire une conscience de l'avenir, et ce en dépit des attentes du spectateur contemporain (qui exige invariablement du divertissement et le reçoit à l'infini), de la civilisation (qui construit des machines de plus en plus perfectionnées, dont nous faisons un usage toujours plus idiot) et de lui-même. Cependant, il n'y a plus d'espoir pour l'avenir de l'humanité : les dés ont déjà été jetés. De ce jeu, il ne reste que le rire, qui peut remplir une fonction astucieusement didactique, et ce sans limite dans le temps ou l'espace. Les premières mondiales d'Une locomotive folle en témoignent (en Allemagne en 1996, en Tchéquie en 2000, aux États-Unis en 2003 et la dernière en Pologne en 2004). Contre tout et même contre lui-même, Witkacy explique invariablement la soi-disant humanité à un homme, à un Être à part, capable de frissons métaphysiques. Il jongle comme un maître avec les conventions, les allusions, les paroles et les gestes pour dévoiler un peu plus les coulisses du Mystère de l'Existence. Et même si elle est dépourvue de thèse, Une locomotive folle a gardé sa capacité de produire des significations, et ce audelà des cultures et des sociétés locales. Après tout, chaque " être civilisé » est un jour monté dans un train, a regardé un thriller "ferroviaire ", a rêvé d'aventure en voyage. Chaque homme a expérimenté la relativité du mouvement et a dû faire face aux autres relativités, dont la plus importante est celle de la valeur. Une locomotive folle est une invitation, adressée à tout un chacun, au voyage à l'encontre des relativités et de l'esprit sain. Et comme toujours chez Witkacy, le rire sauve la pièce d'un moralisme outrancier, puisqu'en fin de compte « le public ne se doutait de rien » 


\section{NOTES}

1. Le texte français de la pièce sera cité d'après l'adaptation de Alain van Crugten in : Stanislaw Ignacy Witkiewicz, Théâtre complet II, L'Âge d'Homme, Lausanne, 2001, pp.95-126. En abrégé : [LF], suivi du numéro de page.

2. J'ai étudié la typologie des personnages dramatiques de Witkacy dont il est question dans l'article: «Tytan Witkacego - Witkacy Tytan »(Le Titan de Witkacy - Witkacy le Titan),in: Pamiętnik Literacki, 2002, cahier 4, pp. 47-50. J'ai exposé ma proposition dans l'article : « Szaleńcy wśród zmechanizowanych bydląt. O bohaterach dramatów Witkacego » (Les fous parmi les bêtes mécanisées. À propos des personnages des drames de Witkacy),in: PamiĘtnik Literacki, 1992, cahier 1, pp. 3-25.

3. Personnage d'une pièce de Witkacy qui a disparu en 1924. (NdT)

4. Degler J., Noty edytorskie (Notes éditoriales), in: Witkiewicz Stanisław Ignacy, Dramaty III (Drames III),oprac. Janusz Degler, PIW, Warszawa, 2004, pp. 830-835.

5. Pour plus d'informations : Ibid., pp. 843-846.

6. Stanisław Ignacy Witkiewicz, Verrückte Lokomotive. Ein Lesebuch mit Bildern des Autors, (Une locomotive folle, anthologie avec illustrations de l'auteur),traduit en allemand parKarl Dedecius, Herausgegeben von Andrzej Wirth, Polnische Bibliothek, Frankfurt am Main, 1985.

7. Ce fut le cas avec la première traduction américaine du texte, faite sur la base du texte dactylographié polycopié : The Crazy Locomotive, traduit en anglais par Daniel C. Gerould, C. S. Dürer, Prude University (Indiana), 1966.

8. Cf. note 1 .

9. Alain van Crugten, « Witkacy i Francuzi » (Witkacy face aux Français), traduit en polonais par Leonia Jabłkowska, in : Teatr, 1975, $\mathrm{n}^{\circ} 11, \mathrm{p} .11$.

10. Avant toute remarque des petits esprits mis en scène, il écrivait: "J'ai vu moi-même l'explosion et l'effondrement d'un édifice dans la pièce de Björnson "Au-delà des forces humaines" au Théâtre de Cracovie. Je sais qu'au point de vue technique c'est exécutable. »: Dramaty III, Op. cit., p. 589. À propos de la mise en scène américaine : Ibid., p. 842.

11. Je considère l'absence de toute mention d'Une locomotive folle comme caractéristique d'une récente étude consacrée à Witkacy (Markowski M. P., Polska literatura nowoczesna. Leśmian, Schulz, Witkacy. [La littérature polonaise moderne. Leśmian, Schulz, Witkacy],Universitas, Cracovie, 2007). Malgré que le chapitre consacré à Witkacy ait clairement une ambition monographique, il omet quelques textes, peut-être en raison du manque de temps et de place (et on a déjà beaucoup écrit sur la Locomotive), peut-être aussi parce que la Locomotive ne cadre pas tout à fait avec cette interprétation. Si nous pouvons dire que la Locomotive s'insère dans une crise de la littérature et que, par rapport à l'épuisement du répertoire de formes et de conventions, il s'agit d'une littérature qui, en s'amusant avec elle-même et en s'auto-parodiant, se fait référence « de biais ". Il est toutefois significatif qu'elle dépasse le jeu de conventions purement littéraires. Elle est tout à la fois, mais pas une « monstrueuse écrivasserie » (voir Markowski M. P., Ibid., pp. 391-392). Elle est une entité extraordinairement compacte dans sa structure, qui sauve Witkacy non seulement d'une "naïve spontanéité ", mais aussi de la scénographie bavarde, ce qui peut expliquer le succès qu'il connaît encore aujourd'hui.

12. Stanisław Witkiewicz, Listy do syna. (Lettres à son fils),oprac. Bożena Danek-Wojnowska, Anna Micińska, PIW, Warszawa,1969, p. 337.

13. Franczak E., Okołowicz S., Przeciw Nicości. Fotografie Stanisława Ignacego Witkiewicza (Contre le néant. Photographie de Stanislaw Ignacy Witkiewicz), Wydawnictwo Literackie, Kraków, 1986. 
14. La première édition de l'essai était internationale, de la même manière que le fonctionnement de la Locomotive dans le monde: "The Crazy Locomotive: Witkacy versus Zola" («Une locomotive folle »: Witkiewicz versus Zola), in: Excavatio, 2001, $\mathrm{n}^{\circ} 1-2$, réédition in: PamiĘtnik Literacki, 2002, cahier 4. Je cite la dernière version du texte, intégrée à la monographie de Tomasik : Ikona nowoczesności - kolej w literaturze polskiej (L'icône de la modernité : le chemin de fer dans la littérature polonaise),Fundacja na Rzecz Nauki Polskiej, Wrocław, 2007, pp. 153-176.

15. Gerould D. C., Stanisław Ignacy Witkiewicz jako pisarz (Stanislaw Ignacy Witkiewicz en tant qu'écrivain), traduit en polonais par Ignacy Sieradzki, PIW, Warszawa, 1981, p. 305, n. 8. Les références à cet ouvrages seront notées en abrégé : [Ger], suivi du numéro de page.

16. Witkiewicz Stanisław Igancy, Pożegnanie jesieni (L'Adieu à l'automne),oprac. Anna Micińska, PIW, Warszawa 1992, p. 114.

17. Voir Tomasik W., Ibid., pp. 162-163. Les références à cet ouvrages seront notées en abrégé : [Tom], suivi du numéro de page.

18. Jan Błoński, Witkacy. Sztukmistrz, filozof, estetyk (Witkacy. Prestidigitateur, philosophe, esthète), Wydawnictwo Literackie, Kraków, 2000, p. 211.

19. Ibid., p. 216.

20. L'(auto)dérision était la manière que Witkacy avait trouvée pour se distancier des procédés modernistes (de la Jeune Pologne). C'était aussi sa façon de surmonter l'inertie de la langue dans le temps, comme disait Sailletan : «Tout a déjà été raconté de bout en bout ». Voir Witkiewicz Stanisław Ignacy, Les cordonniers (trad. de Erik Veaux), in : Théâtre complet II, Op. cit., p. 13.

21. Le premier dit : « No more rum» (trad. : Plus bizarre).C'est signé «Billy Bones » dans L'île au trésor. On peut estimer que c'est probablement plus pervers qu'il n'y paraît. Ce ne sont pas des propos de Billy Bones. Il s'agit d'une inscription de son livre, aussi mystérieux qu'absurde, trouvé par le petit garçon qui est mêlé à la recherche du trésor (voir Robert L. Stevenson, Treasure Island, KGO, Wrocław, 2004, p. 480). Pour le deuxième, il s'agit d'une citation du «Manuel pour les machinistes furieux» de la plume du grand garçon continuellement occupé à imaginer d'excellentes blagues pour son public. Il dit: «VI. Des femmes coupables se tiennent loin des machines; en aucun cas, il ne faut les emmener avec soi dans la locomotive ».

22. C'est un des meilleurs exemples de la «franchise choquante» des héros de Witkacy. Ils sont ici compris, comme l'a conçu Alain van Crugten, «du point de vue de la psychologie des profondeurs et justifiée sur le plan esthétique, (...) les personnages (...) découvrent les motifs internes de leurs initiatives, qui ne sont en rien limités par la moralité conventionnelle ou par les habitudes culturelles » (A. van Crugten, Technika i styl dramaturgii S. I. Witkiewicza [La technique et le style de la dramaturgie de S. I. Witkiewicz],traduit en polonais par T. KĘdzierska. in : PrzeglĄd Humanistyczny, $n^{\circ} 10,1977$, pp. 36-37). Ils ne sont pourtant pas des humains. Alain van Crugten en a écrit plus sur la construction "antipsychologique" des personnages dans sa monographie intitulée S. I. Witkiewicz aux sources d'un théâtre nouveau, Edition L'Âge D'Homme, Lausanne, 1971. À propos des héros d'Une locomotive folle : Cf. Ibid., pp. 254-255.

23. Malgré l'américanisme apparent, ce sont des réminiscences de Demon ruchu (Le démon du mouvement) de Grabiński. Comme l'affirme Błoński (Op. cit,, p. 214), on pourrait voir dans ce nom une allusion au cycle classique de Charles Dickens Mugby Junction (L'embranchement de Mugby).

24. Gerould D. C., Introduction to The Madman and the Nun and Other Plays (Introduction au "Fou et la nonne" et autres pièces), translated and edited by Daniel C. Gerould and C.S. Durer, University of Washington Press, Seattle and London, 1968, p. 81.

25. Dans la traduction de Gerould, nous lisons: "We need two levers to stop the train: one for me and one for my boss. Lend us your rifles" (trad. : Nous avons besoin de deux leviers : un pour moi et un pour mon chef. Prêtez-nous vos fusils)(The Madman..., Ibid., p. 104), alors que la traduction française de Jadwiga Strzałłkowska ressemble à ceci : «Il nous faut deux leviers: à moi et à mon chef pour arrêter le train. C'est le régulateur qui s'est gâté. Donnez vos fusils » (Degler J., Op. cit., p. 548). La version polonaise a par contre été établie par Konstanty Puzyna : „Potrzeba nam dwu 
dźwigni: dla mnie i mego szefa, aby zatrzymać pociĄg. Zepsuł się regulator. Dajcie wasze karabiny" (Witkiewicz S. I., Ibid., p. 584) (Trad. : Il nous faut deux leviers, à moi et à mon chef, pour arrêter le train. C'est le régulateur qui s'est détraqué. Donnez vos fusils » [LF 118].

26. Travaillac est par exemple une «bête qui a sur la conscience au moins trente des assassinats les plus horribles ou plutôt les plus merveilleux »[LF 110].

27. Pas 130 miles à l'heure comme nous lisons dans la traduction de Gerould, bien que celui-ci ait à cet égard respecté les procédés mimétiques de Witkacy, comme il l'explique dans les notes qui accompagnent sa traduction: "we have increased the speed of the train to contemporary equivalents by simply turning kilometers per hour into miles per hour" (The Madman..., Ibid., p. 96) (trad.: Nous avons augmenté la vitesse du train pour qu'elle atteigne l'équivalent de l'époque en convertissant tout simplement les kilomètres à l'heure en miles à l'heure.)

28. Julie a 18 ans, " elle est blonde, très jolie, mais d'une beauté bestiale ». Tengier a par contre « 35 ans, le visage oblong, très expressif. On aperçoit facilement la force de la volonté dans les mâchoires et les arcades sourcilières " [LF 97]. Le fait de donner l'âge exact des personnages en même temps a une autre résonance, il fait non seulement référence à la convention naturaliste mais également à la convention des canards (représentés en Pologne par Fakt), soulignant «l'authenticité » des informations données en précisant l'âge des héros de l'article. Une telle démarche psychologique a justement pour but de rendre les personnages crédibles. Witkacy rend aussi ses personnages crédibles justement en tant que personnages, qui quelques dizaines d'années plus tard auront invariablement 18 et 35 ans.

29. The Madman..., Ibid., p. 107.

30. Et avec elles les conventions de la littérature populaire: "Mais réfléchissez donc "- dit Travaillac à Trefaldi en prenant la décision d'augmenter la vitesse - «nous en causons ainsi ici et là, dans une voiture quelconque de notre train, il y a quelqu'un qui lit la même chose, une histoire semblable tirée de la bibliothèque criminelle de voyage. C'est drôle ! » [LF 109). Comme l'a remarqué Tomasik, cela pourrait par exemple être le tome de nouvelles ferroviaires de Grabiński, que Witkacy ne supportait pas. Il ne supportait pas non plus Karol Irzyskowski, son fervent défenseur, l'auteur de Uroki naturalizmu (Les charmes du naturalisme) (1922).

31. Dans la mesure où le manifeste Nóż $w$ bżuhu (Le couteau dans le ventre), publié à peine un an plus tôt (1922), était caractéristique du futurisme polonais, il a pu faire l'objet de jeux textuels et parodiques de la part Witkacy. La question reste ouverte.

32. George Westinghouse (1846-1914) était en vérité l'inventeur du frein pneumatique pour les trains.

33. Ici je ne peux me référer qu'à l'analyse perspicace de Tomasik (Op. cit., pp. 165-168 et 170-172) et à Franck S. Galassi, Slapstick in Witkiewicz's «Crazy Locomotive» (La farce dans « Une locomotive folle de Witkiewicz), in : The Polish Review, $\mathrm{n}^{\circ} 4,1977$.

34. J'ajouterais aussi : "A notre époque, la piraterie a cessé d'être possible malheureusement ». C'est une allusion au droit à la chute inévitable de la grandeur ainsi qu'à l'impossibilité de revenir aux techniques réalistes, qui a été poussée jusqu'à la perfection par des maîtres tels que Joseph Conrad. C'est Witkacy lui-même qui l'affirme. Le Corsaire était pourtantle roman préféré de Witkacy.

35. J'ai abordé ce sujet dans : Motywy szaleństwa w twórczości Witkacego i Conrada (Les motifs de la folie dans l'œuvre de Witkacy et de Conrad), Uniwersytet Wrocławski, Wrocław, 1999, pp. 52-57.

36. Et nous trouverons, dans le texte de la Locomotive, la «sanction divine " préparée de façon adéquate. La course casse-cou doit être, d'après Travaillac, «quelque chose dans le genre d'un duel » (pour Julie bien sûr) ou « un jugement de Dieu » [LF 109].

37. Il suscite la polémique sur le cinéma, dans laquelle la locomotive «était depuis toujours ». Pour Wikacy, le cinéma était à ses débuts « une copie esclave de la réalité » (voir Degler J., Witkacy $i$ kino. (Witkiewicz et le cinéma)in : Dialog, nº 3, 1996, pp. 131-138. 


\section{RÉSUMÉS}

L'auteure de l'article se penche sur la pièce de Witkacy Une locomotive folle écrits en 1923 et sur originalité et spécificité de cette pièce par rapport à son œuvre et par rapport à son temps. En répondant aux questions : Pourquoi Witkacy place-t-il la machine au centre du drame ? Est-il vraiment contre la mécanisation impitoyablement critiquée de la vie ? En mettant la locomotive sur scène, tourne-il en dérision la Forme Pure dans le théâtre ou salue-t-il ironiquement le théâtre naturaliste ? Marta Skwara analyse en profondeur cette pièce, avec un large appareil scientifique et contexte de théorie de la littérature et l'esthétique. Les noms de Daniel Gerould, Emile Zola, Alain Van Crugten, Wojciech Tomasik, Jan Błoński y apparaissent comme les points de références à côté des constats tout à fait originales et propres à Marta Skwara.

\section{INDEX}

Index géographique : Pologne

oeuvretraite Witkiewicz St. I. : Szalona lokomotywa (Une locomotive folle), Zola É. : Bête

humaine [La]

Index chronologique : entre-deux-guerres, XXe siècle

Mots-clés : littérature polonaise

\section{AUTEURS}

\section{MARTA SKWARA}

Professeur à l'Université de Szczecin, Pologne

Directrice de la section de Littérature comparéemarta.skwara@univ.szczecin.pl 